Special issue of the 3rd International Conference on Computational and Experimental Science and Engineering (ICCESEN 2016)

\title{
Investigation of Buildup Factor in Gamma-Ray Measurement
}

\author{
H. Akyildirim*, F. Waheed, K. GünoĞLu And İ. Akkurt \\ Suleyman Demirel University, Faculty of Arts and Sciences, Department of Physics, Isparta, Turkey
}

\begin{abstract}
Gamma ray measurement is an important issue in nuclear technology, since it is widely used in industry, medicine, agriculture, education research, and some military applications. Gamma ray is also needed to build radiation protection, which is very useful for human health. When gamma radiation penetrates through shielding material, it generates two radiation components within or beyond the shield, namely: the uncollided and the collided photons. Therefore, the buildup factor is an important parameter for gamma ray measurement. Buildup factor is defined as the ratio of the total number of particles at a given point to the number of uncollided particles, at that same point. In this work, we evaluate the gamma-ray buildup factors for copper (Cu-29), as a function of energy, for $0.511,0.662,1.275 \mathrm{MeV}$, by using cesium and sodium radioactive sources. The results show that the value of energy buildup factor decreases with increasing gamma energy, and increases with increasing thickness. Moreover, it was found that at high energies $(1.275 \mathrm{MeV})$, the absorption buildup factor is at minimum when the energy is at high level. The results also reveal that there is no contribution from the scattering photons to the value of buildup factor, in general, at low intensity levels, when the geometry structure is built well. While for bad geometry, the detector measures intensity, which is greater than that described by the main linear attenuation coefficient, because the scattered photons will be detected as well. All in all, in order to get rational results, a well geometry should be used for the future applications.
\end{abstract}

DOI: 10.12693/APhysPolA.132.1203

PACS/topics: Gamma-rays measurement, Radiation protection, Buildup factor, Gamma Spectrometer, Geometry

\section{Introduction}

The backscattering or reflection of photons is a big issue and concern of fundamental importance in radiation shielding, for scientists and developers of gamma rays protection $[1,2]$. Therefore, the design of good geometry is considering as one of the most important issues in this field. Buildup factors, on the other hand, can be obtained from existing tables, developed by the American Nuclear Society. Since these tables are not applicable for a general case, they should be adopted for given kinds of measurements $[3,4]$. Buildup factor is important for proper gamma attenuation measurement and can affect the measurement quality [5]. Gamma ray measurement is required in nuclear technology, since it is used in industry, medicine, agriculture, education, research, and in military applications. It is also required for building of radiation protection, to protect human health $[6,7]$.

As gamma radiation penetrates through shielding material, it produces two radiation components within or beyond the shield, the uncollided photons and the collided photons [8]. Thus, the buildup factor is an important parameter for gamma ray measurement. It is defined as the ratio of the total number of particles at a given point to the number of uncollided particles, at that same point [9].

There are few studies devoted to measurement of the buildup factors. Guvendik [9], has developed a formula of buildup factor for two-layer shields by using point source with the energy from $0.1 \mathrm{MeV}$ to $10 \mathrm{Mev}$. The results

*corresponding author; e-mail: hakanakyildirim@sdu.edu.tr show that values of buildup factors for two-layer materials, obtained with the developed equations agree with the results obtained using MCNP [9].

Kazuo and Hirayama calculated gamma-ray buildup factors up to 40 mean free paths (mfp) for double-layered shields, that had water, concrete and iron, using the EGS4 code, by applying a special splitting technique, at gamma-ray energies of $0.1,0.3,0.6,1,3,6$ and $10 \mathrm{MeV}$. The result has confirmed that the calculation of buildup factor at energy from 1 to $10 \mathrm{MeV}$, using a formula reproduced very well the reference data in most cases [10].

Hopkins [11] has measured and calculated experimentally the linear attenuation coefficient and buildup factor by using four different energies of the $\gamma$-ray photon beam. The results revealed that the buildup factor increased linearly with the attenuator thickness and faster than linearly with the increasing beam energy.

Jubair [12], computed the buildup factor for ceramic materials and sheet of lead, as a standard material, based on gamma ray spectroscopy. The result consisted of the values of mean free path, counts rate per 1000 seconds for good and bad geometry and build up factors, for lead and ceramic materials.

Harima, et al. [13] improved the geometrical progression fitting function for 28 single-material gamma-ray buildup factors to approximate the deep penetration up to $100 \mathrm{mfps}$. The results were tested by the least squares fit and the minimization of maximum fractional deviation. According to the results, the improved geometrical progression formula gave an enhanced fit for all values of buildup factors up to $100 \mathrm{mfps}$, within about $15 \%$. Furthermore, the validity of the geometrical progression parameters was tested and the possibility to obtain a buildup factor for a mixture was demonstrated [13]. 
In this study, the gamma-ray buildup factors were determined for copper. The measurements were performed for $0.511,0.662,1.275 \mathrm{MeV}$ gamma rays using $\mathrm{NaI}(\mathrm{Tl})$ detector.

\section{Experimental details}

\subsection{Preparation of samples}

In order to calculate the buildup factor, three different thicknesses of of pure single-layer copper were used. The weight and thickness of each sample were measured. The prepared samples were tested against gamma-rays using gamma spectrometer at Suleyman Demirel University, Physics Department in the beginning of 2016. The buildup factor and linear attenuation coefficient of the samples were measured for gamma rays of three different energies: $0.511,0.662$, and $1.275 \mathrm{MeV}$. These gamma rays were obtained using ${ }^{22} \mathrm{Na}$ and ${ }^{137} \mathrm{Cs}$ sources. For each thickness, the buildup factor was measured based on the relation between incoming gamma rays and gamma rays penetrated through the material.

\subsection{Gamma spectrometer system}

The gamma spectrometer is used to test whether the material is radioactive and then to find the source of that radioactivity. The gamma spectrometer also identifies the energy of gamma rays of the samples. The gamma spectrometer consists of a detector $(\mathrm{NaI}(\mathrm{Tl})$ in our case), counting electronic system (high voltage source, preamplifier, amplifier 16384-channel multichannel analyser), and a PC employed to record the data. In order to reduce the background level of the system, the detector is shielded using $6 \mathrm{~cm}$ of lead from all sides. Figure 1 shows a schematic description of the spectrometer used in this study. The control of acquisition parameters and analysis of the collected spectra were carried out with aid of MAESTRO-32 (version 6.06) software package.

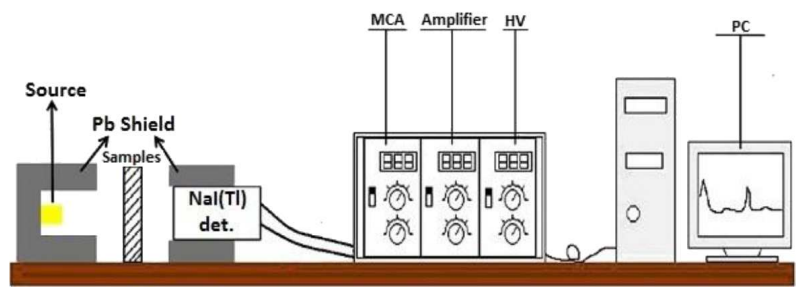

Fig. 1. Schematic view of the experimental setup.

\section{3. $\mathrm{NaI}(\mathrm{Tl})$ detectors}

Scintillation detectors are composed of a scintillator material with the attached photomultiplier tube. When ionizing radiation interacts with the scintillation material, it causes ionization or excitation. Scintillation light, emitted by the phosphor, is collected by photomultiplier tube, it is converted to voltage pulse, as shown in Fig. 2. The amplitude of obtained pulse is proportional to the energy of the radiation. These detectors can also be used for counting and energy discrimination.

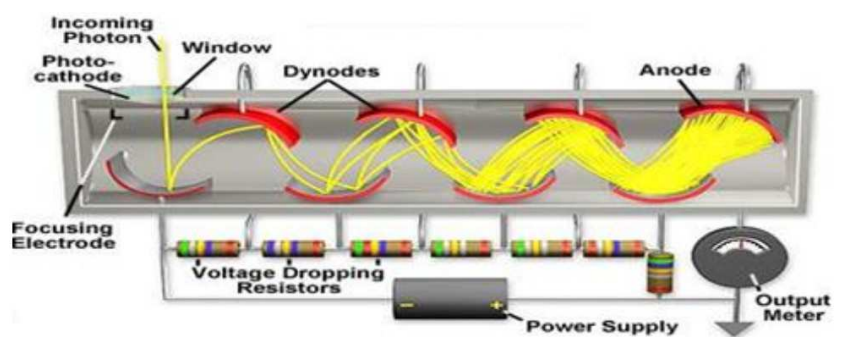

Fig. 2. Photomultiplier tube of a scintillation detector.

\subsection{Geometry}

The measurement system consists of a measuring head, including a source container, with ${ }^{22} \mathrm{Na}$ and ${ }^{137} \mathrm{Cs}$ sources, of $37 \mathrm{kBq}$ for each source, and a scintillation detector. Three sample plates with thicknesses of $0.09,0.18$ and $0.227 \mathrm{~cm}$, with sizes of $9.5 \times 9.5 \mathrm{~cm}^{2}$ were used.

The distance between the detector and the source was about $9.5 \mathrm{~cm}$. The gamma-ray beam was collimated. The diameter of the collimator was approximately $4.5 \mathrm{~cm}$ at the measurement point and about $5 \mathrm{~cm}$ at the detection point. The general configuration of the geometry is shown in Fig. 3.

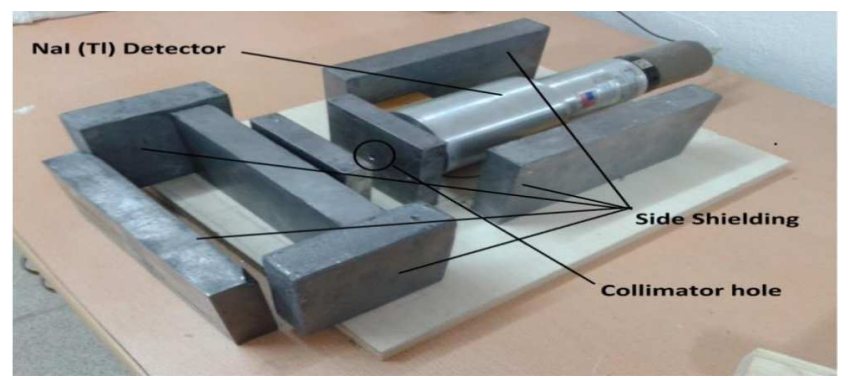

Fig. 3. Buildup factor measurement geometry.

To eliminate or to reduce the scattered photons and the background activity, lead plates were used to cover the source and the detector, as shown in Fig. 4.

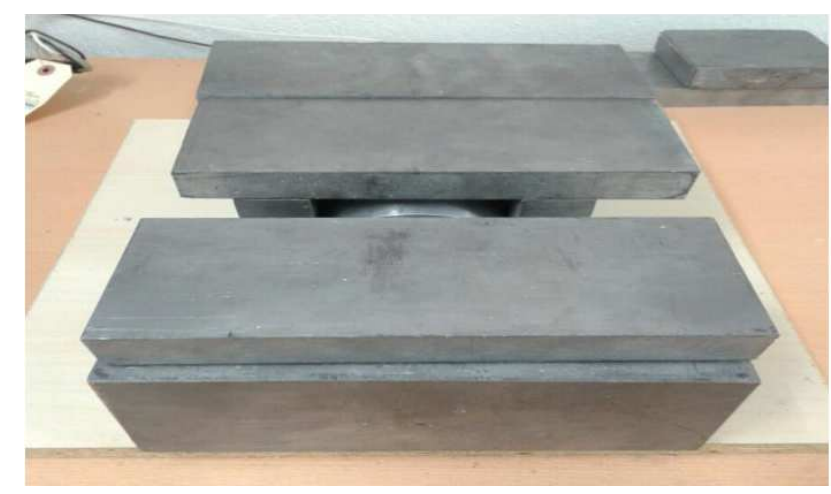

Fig. 4. Side shielding plate. 


\subsection{The buildup factor measurement}

The buildup factor is measured based on the relation between the incoming gamma rays and gamma rays passing through the materials, as shown in Fig. 5. If the initial intensity of gamma rays is $I_{0}$, the buildup factor $B$ is calculated according to the following standard equations:

$$
\begin{aligned}
& I=B I_{0} \mathrm{e}^{-\mu x}, \\
& B=I / I_{0} \mathrm{e}^{\mu x},
\end{aligned}
$$

where $I$ is the measured detector count with the absorber of thickness $x(\mathrm{~cm})$, and $I_{0}$ is measured count without the absorber.

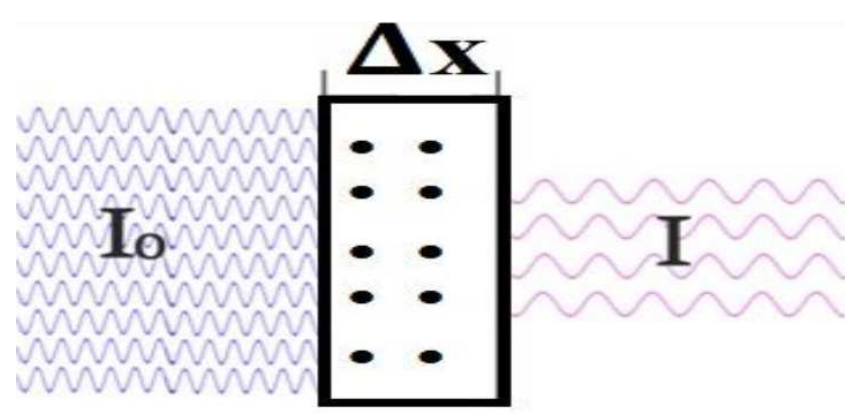

Fig. 5. Penetration of radiation through the absorber.

Plotting $I_{0} / I$ versus $x$ in a natural logarithm scale shows the quality of the fit $R^{2}$. The intensity of incoming $I_{0}$ and penetrated $I$ gamma rays are obtained from energy spectrum.

\section{Results and discussion}

In this study, the objective is to compute the energy absorption and the buildup factors. Three different copper plates of different thicknesses were prepared as absorbers. The results were obtained using gamma spectroscopy and live radioactive sources of ${ }^{22} \mathrm{Na}$ and ${ }^{137} \mathrm{Cs}$ at gamma energies of $0.511,0.662$, and $1.275 \mathrm{MeV}$. Figures $6-8$ show the absorption buildup factors as functions of thickness for different values of gamma energy.

It can be concluded that the buildup factor increases with increasing thickness of the attenuator. When thickness increases, the probability of occurrence of an uncollided photon decreases.

Figure 9 shows the buildup factor of copper is as a function of energy for the attenuator thickness of $0.2 \mathrm{~cm}$. It is seen that the buildup factor decreases with the increasing energy, for the constant thickness of the attenuator. When gamma energy increases, the probability of photon collision decreases and the number of scattered photons decrease, which leads to the decrease of the buildup factor.

As it is obvious from Fig. 10, buildup factor is a function of the energy and the thickness of the attenuator.

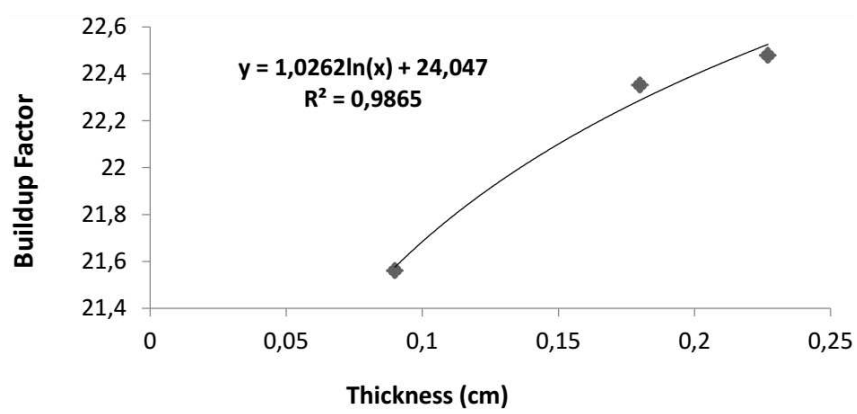

Fig. 6. Copper buildup factor for the energy of $0.511 \mathrm{MeV}$.

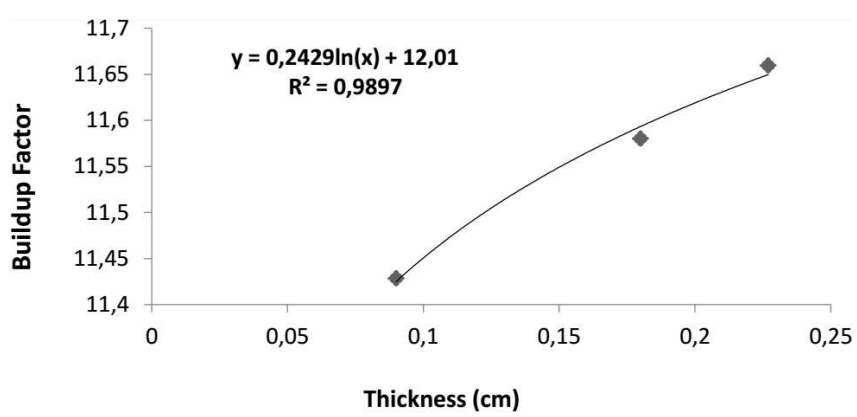

Fig. 7. Copper buildup factor for the energy of $0.662 \mathrm{Mev}$

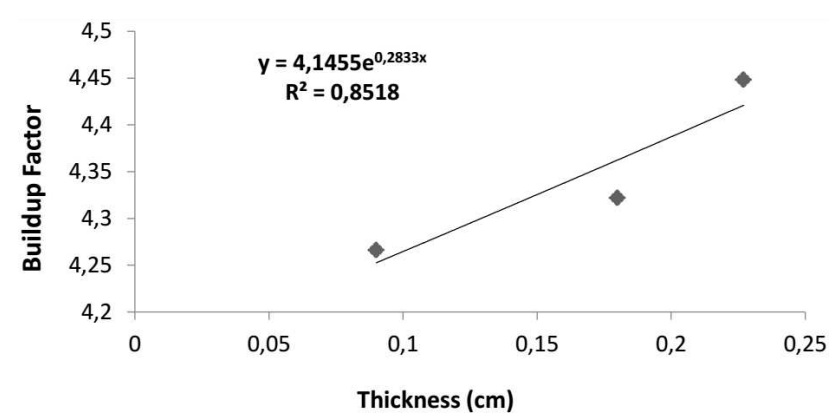

Fig. 8. Copper buildup factor for the energy of $1.275 \mathrm{MeV}$.

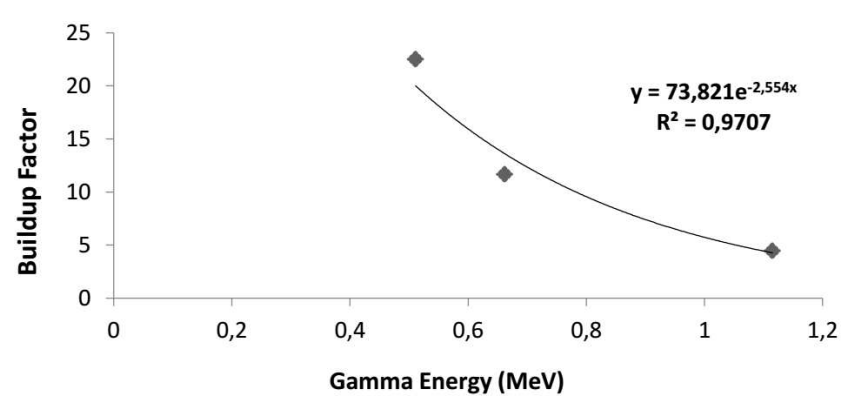

Fig. 9. Copper buildup factor as a function of gamma energy for the attenuator thickness of $0.2 \mathrm{~cm}$. 


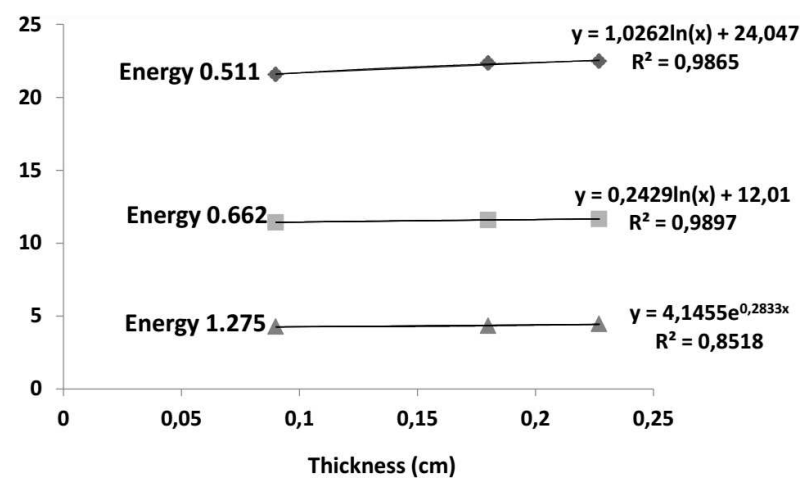

Fig. 10. Copper buildup factor as a function of energy and thickness.

From this figure we can conclude that the buildup factor decreases with increasing energy for the same thickness of attenuator and it increase with the increase of thickness, for the same gamma energy.

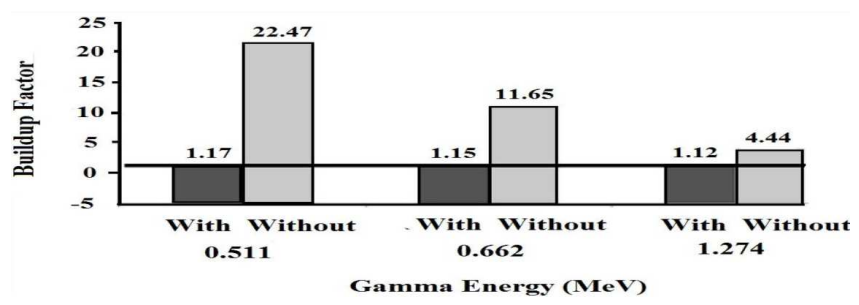

Fig. 11. Copper buildup factor with and without the collimator.

Figure 11 illustrates the variation of the value of buildup factor for copper for two cases: with and without the collimator. From these result it can be concluded that we can get a good result for good geometry.

\section{Conclusions}

Since the buildup factors for a general case, that can be applied directly, are not available, they should be developed and calculated for any specific case. Measurement of these factors, therefore, will contribute to the measurement quality and the employed system is an important proper gamma attenuation measurement system.
In this work three pure copper absorber samples were produced. These produced materials were examined against gamma-rays. The measurements were carried out for gamma rays with $0.511,0.662$, and $1.275 \mathrm{MeV}$, using $\mathrm{NaI}(\mathrm{Tl})$ detector. The measurements show that the value of buildup factor increases with increasing thickness at a given gamma energy, and it decreases with the increasing gamma energy at a given thickness of material.

When the geometry is good and there are no scattered photons, the value of buildup factor is in general at low level. On the other hand, for bad geometry the intensity, measured by the detector, is greater than that described by main linear attenuation coefficient, since the scattered photons are also detected. This leads to a conclusion, that a good geometry, obtained in this study, should be applied in the future measurements in order to get more plausible results.

\section{References}

[1] G.S. Brar, S. Sidhug, P.S. Singh, G.S. Mudahar, Radiat. Phys. Chem. 49, 977 (1998).

[2] R.G. Jaeger, A.B. Chilton, E.P. Blizard, A. Honig, A. Jaeger, H.H. Eisenlohr, Engineering Compendium on Radiation Shielding, Vol. I, New York Inc., 1968.

[3] E. Kavas, N. Ekinci, Asian J. Chem. 28, 1673 (2016).

[4] S.R. Manohara, S.M. Hanagodimath, L. Gerward, K.C. Mittal, J. Korean Phys. Soc. 59, 2039 (2010).

[5] A. Shimizu, H. Hirayama, J. Nucl. Sci. Tech. 40, $192(2003)$.

[6] A. Shirani, M.H. Alamatsaz, Iranian J. Sci. Technol. A1, 29 (2013).

[7] B. Singh, V. Kumar, M. Devi, S. Sidhug, Int. J. Latest Res. Sci. Technol. 2, 73 (2013).

[8] S. Singh, S. Ghumman, C. Singh, S. Thind, S. Mudahar, Ann. Nucl. Energy 37, 681 (2010).

[9] M. Guvendik, Ph.D. Thesis, University of MissouriRolla, 1999.

[10] S. Kazuo, H. Hirayama, Radiat. Phys. Chem. 64 583 (2001).

[11] D.N. Hopkins, Determination of the linear attenuation coefficients and buildup factors of MCP-96 alloy for use in tissue compensation and radiation protection, Diss. Ball State University, 2010.

[12] S.I. Jubair, Iraqi J. Appl. Phys. 7, 23 (2011).

[13] Y. Harima, N. Kurosawa, Y. Sakamoto, Progr. Nucl. Sci. Technol. 4, 548 (2014). 